\title{
Case Report: Acute-on-Chronic Liver Failure: Making the Diagnosis between Infection and Acute Alcoholic Hepatitis
}

\author{
Carmen Sendra, MD ${ }^{1}$ Javier Ampuero, MD, PhD ${ }^{1,2}$ Álvaro Giráldez Gallego, MD ${ }^{1}$ \\ José Manuel Sousa, MD ${ }^{1}$ María Serrano Jiménez, $\mathrm{MD}^{3}$ Manuel Romero-Gómez, MD, $\mathrm{PhD}^{1,2}$
}

${ }^{1}$ Inter-Centre Unit of Digestive Diseases and CIBERehd, Virgen
Macarena - Virgen del Rocío University Hospitals, University of Sevilla,
Sevilla, Spain
2 Instituto de Biomedicina de Sevilla, Spain
${ }^{3}$ Pathology Unit, Virgen del Rocio University Hospital, Sevilla, Spain

Address for correspondence Javier Ampuero, MD, PhD, Unit for the Clinical Management of Digestive Diseases, Virgen Macarena - Virgen del Rocío University Hospitals, Sevilla, Spain (e-mail: javi.ampuero@gmail.com).

Semin Liver Dis 2016;36:181-186.

\begin{abstract}
Keywords

- acute-on-chronic liver failure

- organ failure

- systemic inflammation

- infection

- acute alcoholic hepatitis

Acute-on-chronic liver failure (ACLF) represents a reversible syndrome associated with high short-term mortality, characterized by acute decompensation in patients with chronic liver disease and extrahepatic organ failure. Diagnosis and prognosis assessment is based on a newly developed diagnostic score, the Chronic Liver Failure Consortium Organ Failure score. Susceptibility to infections and systemic inflammation are typical triggers. The authors report a case in which a patient with alcohol-related cirrhosis was admitted to the hospital with acute decompensation and developed ACLF during hospitalization. This case led to an evaluation of the underlying process causing ACLF: infection versus acute alcoholic hepatitis.
\end{abstract}

Acute-on-chronic liver failure (ACLF) is a newly recognized syndrome associated with a high mortality. The prevalence of ACLF is around $30 \%$ among patients hospitalized with acute decompensation; it is more frequent in alcoholic- and hepatitis-B-associated cirrhosis. ${ }^{1}$ Clinical course and number of organ failures are the most relevant factors associated with ACLF-related mortality, irrespective of triggering factors. ${ }^{2}$

We report a case of a patient admitted with acute decompensation secondary to infection and/or severe acute alcoholic hepatitis (AAH), who developed ACLF-1 during hospitalization. The patient was successfully treated with an antibiotic and steroid course in sequence.

\section{Case Presentation}

A 48-year-old man, showing a compensated alcohol-related cirrhosis since 2011 (Child-Pugh A5), was admitted to the hospital in October 2015 because of jaundice and unspecific abdominal pain. His alcohol intake exceeded $80 \mathrm{~g} / \mathrm{d}$ until
2009; it had decreased to $40 \mathrm{~g} / \mathrm{d}$ at the time of the admission. In 2012, he underwent an upper endoscopy, which detected small esophageal varices. The patient had neither previous complications of cirrhosis nor current treatment. One week prior to the admission, he had had cough and fever (up to $40^{\circ} \mathrm{C}$ ), and was treated with acetaminophen $500 \mathrm{mg} 3$ times per day. The patient denied changing the pattern of alcohol consumption or taking illicit or recreational drugs. Physical examination revealed jaundice, spiders on the trunk, and no signs of hepatic encephalopathy, clinical ascites, or edema. Vital signs, including peripheral capillary oxygen saturation (98\%) and blood pressure (135/65 mm $\mathrm{Hg}$ ), were normal. Laboratory tests showed a high total bilirubin (TB) level $(25.41 \mathrm{mg} / \mathrm{dL})$ with typical alcohol-related liver enzymes values (alanine aminotransferase $80 \mathrm{IU} / \mathrm{mL}$, alanine aminotransferase $40 \mathrm{IU} / \mathrm{mL}$ ), as well as international normalized ratio $(1.53)$ and platelet count $\left(97 \times 10^{9} / \mathrm{L}\right)$ linked to advanced liver disease. By contrast, kidney function was normal (creatinine $0.54 \mathrm{mg} / \mathrm{dL}$ ). In addition, the abdominal
Issue Theme Acute-on-Chronic Liver Disease; Guest Editor, Rajiv Jalan, MD
Copyright $\odot 2016$ by Thieme Medical Publishers, Inc., 333 Seventh Avenue, New York, NY 10001, USA. Tel: +1(212) 584-4662.
DOI http://dx.doi.org/ 10.1055/s-0036-1583203. ISSN 0272-8087. 
182 Making the Diagnosis between Infection and Acute Alcoholic Hepatitis Sendra et al.

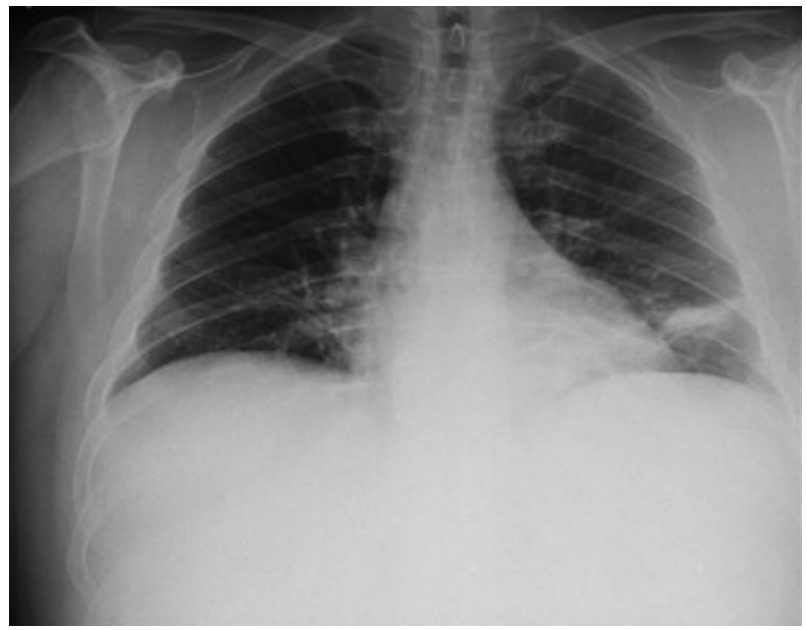

Fig. 1 Chest X-ray showing a condensation in the left lower lobe.

ultrasound showed minimal perihepatic ascites and the chest X-ray a pneumonic process (-Fig. 1).

Empirical antibiotic therapy with ceftriaxone (2 g intravenously [IV] once per day) and levofloxacin (500 mg IV once per day) was started to treat pneumonia as the triggering factor of decompensated cirrhosis (the patient did not meet ACLF criteria) ( - Fig. 2A). Pentoxifylline 400 mg orally 3 times per day was also added to the treatment. Upper endoscopy showed five enlarged veins, three of them with protruding vessels, and a moderate portal hypertensive gastropathy. Consequently, carvedilol $6.25 \mathrm{mg}$ orally twice per day was prescribed. Eleven days after admission, he was asymptomatic although with similar TB $(21.57 \mathrm{mg} / \mathrm{dL})$. However, hypotension and an acute kidney injury (creatinine $1.75 \mathrm{mg} / \mathrm{dL}$ ) were observed. At that moment, he met ACLF-1 criteria
(-Fig. 2B). Given the temporal relation, carvedilol was withdrawn and albumin IV was prescribed ( $1 \mathrm{~g} / \mathrm{kg} / \mathrm{d}$ for 3 days). Moreover, a diagnostic paracentesis was performed, ruling out spontaneous bacterial peritonitis (SBP). In a computed tomography scan, a partially resolved pneumonic process was observed after a 15-day period of antibiotic treatment (-Fig. 3). However, there was a worsening of the overall status, with increased abdominal pain. A glycopeptide-susceptible Enterococcus faecium was detected in an ascitic fluid culture from the previous diagnostic paracentesis. Therefore, the patient suffered from a symptomatic monomicrobial nonneutrocytic bacterascites. We replaced levofloxacin with teicoplanin $400 \mathrm{mg}$ IV once per day after consulting with the infectious diseases department (and keeping ceftriaxone). There was an improvement in the patient's overall status and a normalization of kidney function after a 5-day period of ceftriaxone and teicoplanin. Nevertheless, bilirubin levels were similar to previous days (TB $26.82 \mathrm{mg} / \mathrm{dL}$ ), so we decided to perform a transjugular liver biopsy. Histological findings showed AAH on alcoholic liver cirrhosis (-Fig. 4), and the hepatic venous pressure gradient was $27 \mathrm{~mm} \mathrm{Hg}$. After normalization of kidney function, propranolol $20 \mathrm{mg}$ orally twice per day was administrated, but it had to be withdrawn because of hypotension and a mild acute kidney injury, which resolved a few days later (-Fig. 5A).

One month after admission and one week after the end of treatment for both pneumonia and bacterascites, prednisone $40 \mathrm{mg}$ orally once per day was added to the treatment, according to histological AAH findings and a Maddrey score $>$ 32. The patient had TB $18.49 \mathrm{mg} / \mathrm{dL}$ and prothrombin time $17.6 \mathrm{~s}$ at the beginning of the steroid cycle. He was discharged with a 1-week course of prednisone after reducing TB levels

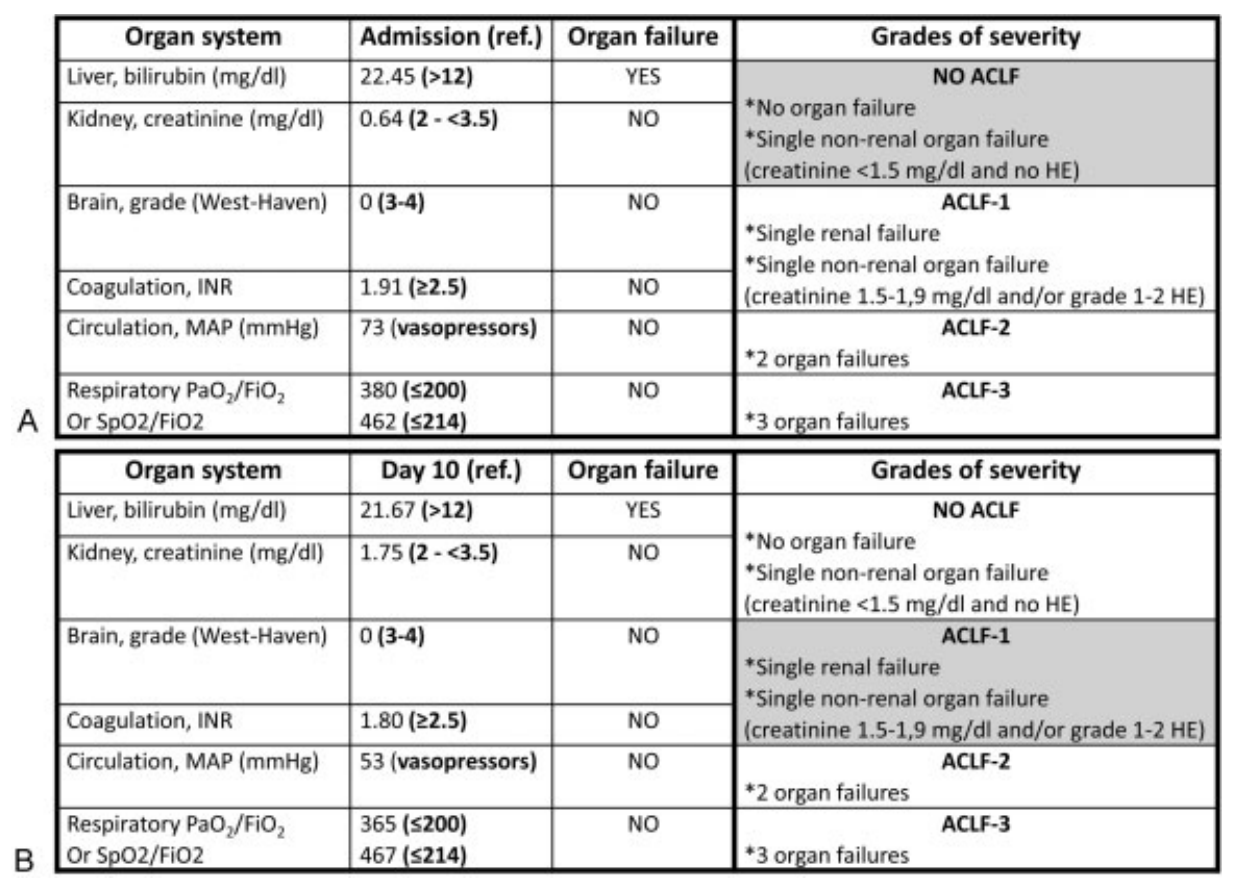

Fig. 2 (A) The Chronic Liver Failure Consortium organ failure score. (B) Grades of severity of acute-on-chronic liver failure. ACLF, acute-on-chronic liver failure; MAP, mean arterial pressure; INR, international normalized ratio; HE, hepatic encephalopathy. 

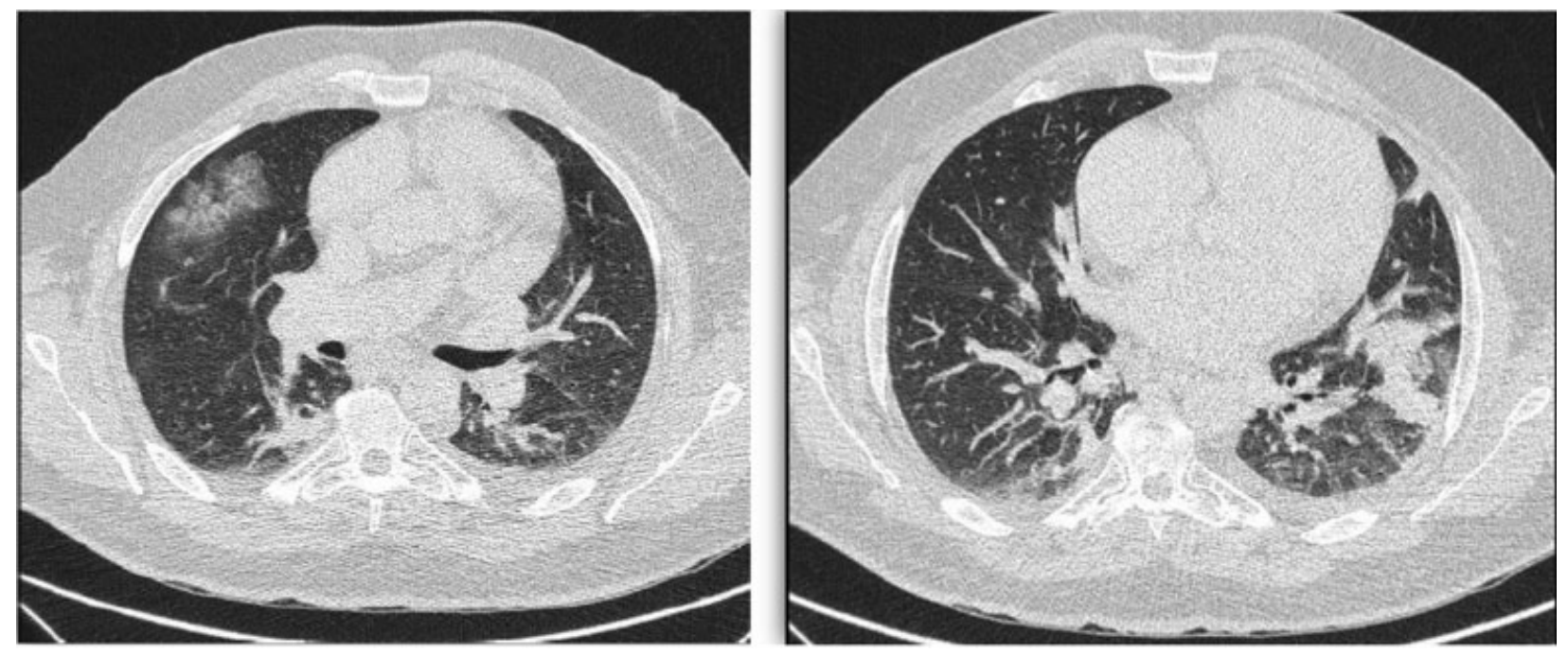

Fig. 3 Chest computed tomography scan showing bilateral atelectasis and a partially resolved pneumonic process.

(10.25 mg/dL) (- Fig. 5B). When the patient finished a 28-day period of treatment with prednisone $40 \mathrm{mg}$ daily followed by a 2-week taper, he developed a cellulitis affecting his right leg. He was treated with amoxicillin-clavulanic acid (500/125 mg orally 3 times per day for 8 days) and diuretics until resolution (-Fig. 6). Simultaneously, laboratory testing showed a decrease in TB levels to $6.04 \mathrm{mg} / \mathrm{dL}$.

\section{Discussion}

Acute-on-chronic liver failure is an increasingly recognized entity characterized by acute deterioration in liver function in a patient with chronic liver disease, together with organ failure and a high short-term mortality. ${ }^{3}$ Over time, several diagnostic criteria have been proposed for this syndrome. The CANONIC (CLIF Acute on Chronic Liver Failure) study provided a solid definition of the epidemiology, diagnosis, and prognosis of ACLF based on prospective data to identify patients with a 28 -day mortality over $15 \%{ }^{4}$ The Chronic Liver Failure Consortium Organ Failure (CLIF-C-OF) score, derived from Sequential Organ Failure Assessment score criteria, determines the prognosis depending on the specific organ involved. Thus, patients are classified as having ACLF with three grades of severity, with an increasing risk of short-term death from grade $1(22 \%)$ to grade $3(77 \%){ }^{5}$

The development of ACLF may occur at any time during hospitalization in patients with compensated to longstanding cirrhosis. About $10 \%$ of patients admitted with decompensated cirrhosis will develop ACLF, as shown in this case report. In fact, our patient showed only liver failure at admission, which could not be considered ACLF. However, the patient reached ACLF-1 when acute kidney injury presented at day 10. It has been reported that ACLF grades between the third and seventh day after diagnosis (d3-d7 ACLF) predicted the short- and mid-term prognosis more accurately than the initial grade. Regarding ACLF-1, most patients will experience the resolution of the syndrome, similar to the current clinical case. Therefore, the dynamic nature of this syndrome should make us be alert because patients can move rapidly from any grade to ACLF to its resolution.

A precipitating event is frequently unnoticed and not necessary for diagnosis. Bacterial infection is the main identified insult (33\%), followed by active alcoholism. ${ }^{1}$ Among infections, SBP, pneumonia, and urinary tract infections are the most relevant. The pathophysiology of infection-induced organ failure remains poorly understood, but a rapid deterioration of cardiovascular function and organ perfusion, as well as direct organ damage secondary to an exaggerated inflammatory response appear to be essential. ${ }^{6}$ Our patient exhibited two of the main infections (pneumonia and bacterascites) typically associated with ACLF. Initially, we started antibiotic treatment for pneumonia as the main trigger of the acute decompensation, and, then bacterascites was properly treated. $^{7}$

The systemic inflammatory response is a typical feature of ACLF. Thus, the increased white cell count at admission is an independent predictor of mortality, representing a nonspecific response to inflammation whose underlying mechanism is unknown. ${ }^{8}$ The CLIF-C-ACLF score was designed to predict survival, which results from a combination of three factors independently associated with mortality: CLIF-C-OF score, age, and white cell count. This score, by measuring both hepatic and extrahepatic organ dysfunction, discriminates significantly better between survivors and nonsurvivors than does the Model of End-Stage Liver Disease (MELD) and the Child-Pugh scoring systems, reflecting the impact of organ failure on prognosis. ${ }^{9}$

On the other hand, the recent study by Mookerjee et al showed that ongoing treatment with nonselective $\beta$ blockers (NSBBs) was safe and independently associated with improved 28-day survival by reducing the severity of ACLF and preventing its progression to more advanced stages. ${ }^{10}$ The mechanism appears to be related to their beneficial effects on gut motility and permeability, reducing bacterial translocation and systemic inflammation. However, our patient developed ACLF- 1 because of the addition of kidney injury to liver failure 


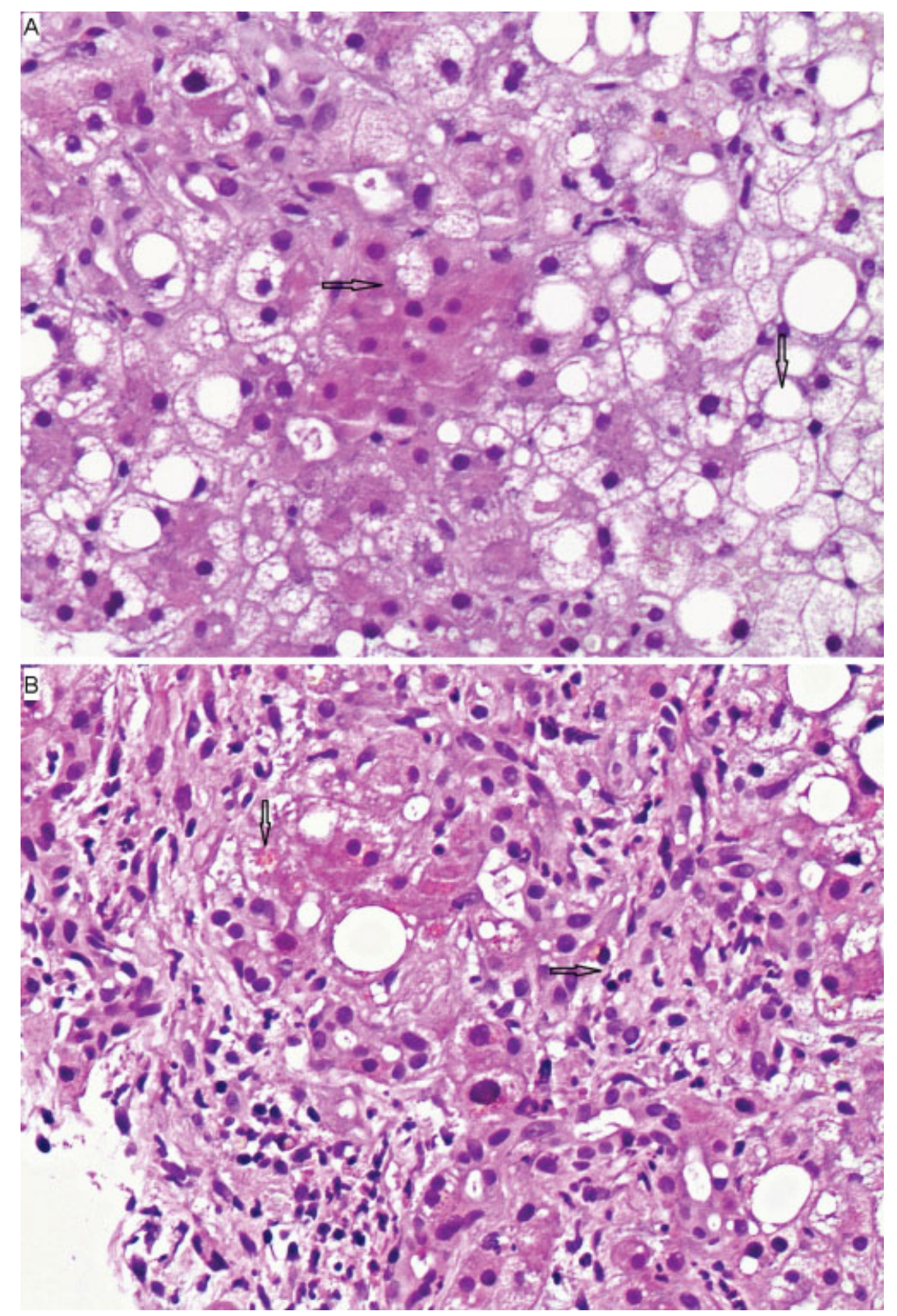

Fig. 4 Liver biopsy consistent with acute alcoholic hepatitis. (A) Numerous slightly enlarged hepatocytes (ballooning) with moderate macrovesicular fatty change (vertical arrow); several cells containing intracytoplasmic aggregates of amorphous, eosinophilic material, so-called Mallory's hyaline (horizontal arrow) (hematoxylin and eosin [H\&E], $\times 20$ ). (B) Occasional mild intracytoplasmic cholestasis (vertical arrow) and focal necrosis accompanied by a mixed inflammatory cellular infiltrate with elevated number of neutrophils (horizontal arrow) (H\&E, $\times 20)$.

in the setting of administration of carvedilol. This aspect improved after NSBB withdrawal and infusion with IV albumin. ${ }^{11,12}$

Our patient showed high TB levels despite an observational period since both infectious processes were solved. Given the concomitant presence of biopsy-proven AAH, we added prednisone $40 \mathrm{mg}$ orally to the treatment (in combination with pentoxifylline started at admission), observing a progressive decrease in TB levels until discharge. ${ }^{13}$ Whether this latter condition was the result from the steroid course or from the time-dependent evolution of the liver failure is impossible to determine. Regarding AAH, controversy persists about steroid use. ${ }^{14}$ In this setting, a major drawback of steroid therapy is the increased susceptibility to infections, as confirmed by the lower leg cellulitis in our patient. The recent study by Thursz et al showed mortality attributed to infection was similar regardless of whether prednisolone was administered. $^{15}$

In conclusion, the early diagnosis and treatment of precipitating events in an enhanced or critical care environment results in higher survival rates for patients suffering from ACLF. However, more than one precipitant factor requiring treatment could be noted in this entity, making the diagnosis sometimes difficult. Acute-on-chronic liver failure is a dynamic syndrome that can vary during the hospitalization, requiring a routine evaluation of its presence. 

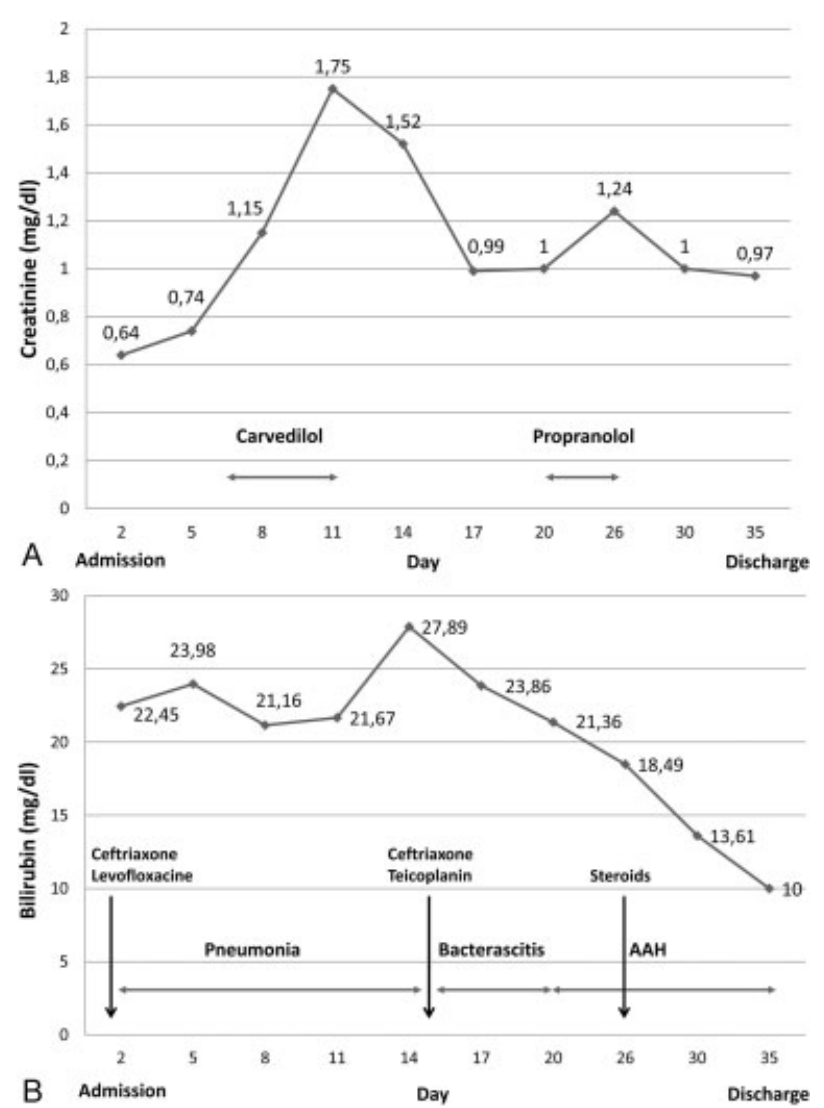

Fig. 5 Laboratory tests during hospitalization. (A) Creatinine levels. (B) Bilirubin levels. AAH, acute alcoholic hepatitis.

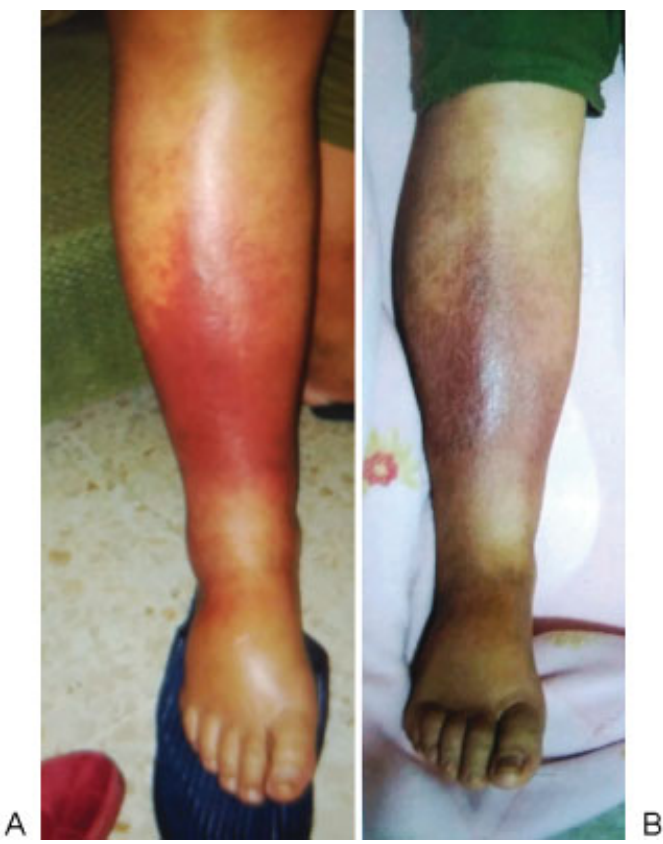

Fig. 6 Right leg cellulitis after steroids treatment (A) and after an 8day treatment with oral diuretics and amoxicillin-clavulanic acid (B).

\section{Abbreviations}

AAH acute alcoholic hepatitis

ACLF acute-on-chronic liver failure

NSBBs nonselective beta blockers

SBP spontaneous bacterial peritonitis

TB total bilirubin

\section{References}

1 Arroyo V, Moreau R, Jalan R, Ginès P; EASL-CLIF Consortium CANONIC Study. Acute-on-chronic liver failure: a new syndrome that will re-classify cirrhosis. J Hepatol 2015;62(1, Suppl): S131-S143

2 Gustot T, Fernandez J, García E, et al; CANONIC Study Investigators of the EASL-CLIF Consortium. Clinical course of acute-on-chronic liver failure syndrome and effects on prognosis. Hepatology 2015; 62(1):243-252

3 Jalan R, Ginès P, Olson JC, et al. Acute-on chronic liver failure. J Hepatol 2012;57(6):1336-1348

4 Bernal W, Jalan R, Quaglia A, Simpson K, Wendon J, Burroughs A. Acute-on-chronic liver failure. Lancet 2015;386(10003): 1576-1587

5 Moreau R, Jalan R, Gines P, et al; CANONIC Study Investigators of the EASL-CLIF Consortium. Acute-on-chronic liver failure is a distinct syndrome that develops in patients with acute decompensation of cirrhosis. Gastroenterology 2013;144(7):1426-1437, 1437.e1-1437.e9

6 Jalan R, Fernandez J, Wiest R, et al. Bacterial infections in cirrhosis: a position statement based on the EASL Special Conference 2013. J Hepatol 2014;60(6):1310-1324

7 Runyon BA. Monomicrobial nonneutrocytic bacterascites: a variant of spontaneous bacterial peritonitis. Hepatology 1990; 12(4 Pt 1):710-715

8 Katoonizadeh A, Laleman W, Verslype C, et al. Early features of acute-on-chronic alcoholic liver failure: a prospective cohort study. Gut 2010;59(11):1561-1569

9 Jalan R, Saliba F, Pavesi M, et al; CANONIC study investigators of the EASL-CLIF Consortium. Development and validation of a prognostic score to predict mortality in patients with acute-on-chronic liver failure. J Hepatol 2014;61(5):1038-1047

10 Mookerjee RP, Pavesi M, Thomsen KL, et al. Treatment with nonselective beta blockers is associated with reduced severity of systemic inflammation and improved survival of patients with acute-on-chronic liver failure. J Hepatol 2016;64(3):574-582

11 Mandorfer M, Bota S, Schwabl P, et al. Nonselective $\beta$ blockers increase risk for hepatorenal syndrome and death in patients with cirrhosis and spontaneous bacterial peritonitis. Gastroenterology 2014;146(7):1680-90.e1

12 Sersté T, Njimi H, Degré D, et al. The use of beta-blockers is associated with the occurrence of acute kidney injury in severe alcoholic hepatitis. Liver Int 2015;35(8):1974-1982

13 European Association for the Study of Liver. EASL clinical practical guidelines: management of alcoholic liver disease. J Hepatol 2012; 57(2):399-420

14 Mathurin P, O'Grady J, Carithers RL, et al. Corticosteroids improve short-term survival in patients with severe alcoholic hepatitis: meta-analysis of individual patient data. Gut 2011;60(2):255-260

15 Thursz MR, Richardson P, Allison M, et al; STOPAH Trial. Prednisolone or pentoxifylline for alcoholic hepatitis. N Engl J Med 2015; 372(17):1619-1628 